\title{
Single-phase Photovoltaic Grid-connected Inverter Current Control Method Based on MRAC
}

\author{
ZHANG Ruiping ${ }^{1,2}$ LU Hongxia ${ }^{1}$ Yang Jie $^{1}$ ZHANG Tingrong ${ }^{1}$ \\ ${ }^{1}$ School of Automation and Electrical Engineering, Lanzhou Jiaotong University, \\ Lanzhou 730070, Gansu Province, China. \\ ${ }^{2}$ Rail Transit Electrical Automation Engineering Laboratory of Gansu Province, \\ Lanzhou Jiaotong University, Lanzhou 730070,Gansu Province, China
}

\begin{abstract}
Keywords:photovoltaic, grid-connected converter, MIT adaptive law, model reference adaptive control(MRAC)
\end{abstract}

\begin{abstract}
In view of that PI controller parameters changeswhen system model parameters of single-phase photovoltaic inverter change, a gradient reference based adaptive current loop control method is proposed. Ignoring the high order harmonics in PWM inverter, the mathematical model of the grid connected system is deduced, the reference model is selected reasonably,me according to the change parameters by using the normalized MIT adaptive law.The grid-connected current has good tracking effect on the instruction current. Control effect is insensitive to the change of the filter parameters, and has fast tracking speed and strong robustness.
\end{abstract}

\section{Preface}

With the intensification of energy crisis and environmental pollution, more and more countries pay attention to the application of clean energy such as solar energy and wind power, and it becomesa major issue in the development of new energy at home and abroad ${ }^{[1,2]}$. The single stage $\mathrm{PV}$ (PhotoVoltaic) grid connected power system is composed of DC/AC converter and its control part. The controller performs the maximum power tracking and the injected grid current regulating respectively. As shown in Figure 1, two-stage PV grid-connected power generation system consists of two parts ${ }^{[3]}$, DC/DC conversion and maximum power tracking control, DC/AC inverter and grid-connected control $^{[3]}$. The DC / DC converter is used to boost the DC voltage and to make the PV array output maximum power by adjusting the dutyin real time ${ }^{[4]}$. DC/AC inverter and its control part is used to stabilize the DC voltage and control the network side current ${ }^{[5]}$.

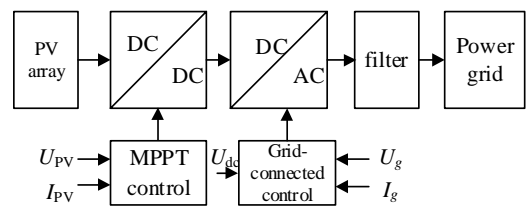

Fig.1 Structure block diagram of photovoltaic power generation system

The PV grid-connected inverter is consists of inverter, filter circuit and control system. The filter circuit generally has L, LC and LCL, and inductance filter is often used in small capacity inverter systems. The topological structure of single-phase PV grid-connected inverter is shown in FIG. 2. 
The voltage loop is used for stabilizing the DC side voltage and generating a command signal of the grid-connected current, and the current loop is used for controlling the grid-connected current, realizing the synchronization of the grid-connected current and the grid voltage and tracking the command current.

The current loop of inverter is generally consist of hysteresis loop control ${ }^{[5]}$ and fixed-frequency control based on PWM modulation ${ }^{[6,7]}$. In order to improve the accuracy of hysteresis control, it is necessary to reduce the hysteresis loop width, and the switching frequency of the inverter will increase drastically. PI control often adopts PWM modulation ${ }^{[8]}$. Design of the PI controller parameter is related to the parameters of the controlled object model. If the filter inductance, the modulation ratio and the inverter parameters change during system operation, the PI parameters need to be readjusted. This paper proposes a model reference adaptive control(MRAC) based on gradient method which is applicable to time-varying system. The controller parameters can be adjusted in real time according to the change parameters ${ }^{[9]}$.

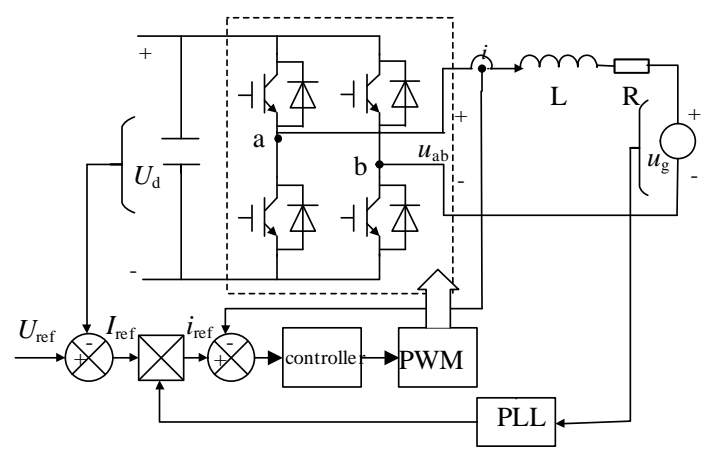

Fig.2 Control block diagram of grid connected inverter

\section{Mathematical Model of Grid-connected Inverter}

The AC side of the inverter is connected to the power grid through inductive filtering, and the voltage equation of the $\mathrm{AC}$ side is

$$
u_{\mathrm{ab}}(t)=L \frac{\mathrm{d} i(t)}{\mathrm{d} t}+R i(t)+u_{\mathrm{g}}(t)
$$

To make the Laplace transform of both sides of the formula (1), there are:

$$
G_{1}(s)=\frac{I(s)}{U_{\mathrm{ab}}(s)-U_{\mathrm{g}}(s)}=\frac{1}{L s+R} .
$$

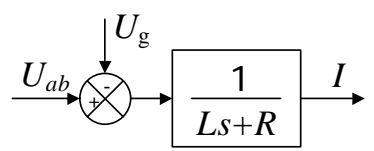

Fig1.3 Filter block diagram

Ignoring higher harmonics, PWM inverter can be seen as a proportional delay link. 


$$
G_{2}(s)=\frac{U_{\mathrm{ab}}(s)}{U_{m}(s)}=k_{\mathrm{PWM}} \mathrm{e}^{-T s}
$$

In the formula, $T$ is the switching cycle of the inverter, $k_{\mathrm{PWM}}=m U_{\mathrm{d}}$, and $m$ is PWM modulation ratio. When the inverter switching frequency is higher, the period is smaller. Equation (3) can be approximated by first-order inertia.

$$
G_{2}(s) \approx k_{\mathrm{PWM}} \frac{1}{1+T s}
$$

$$
\stackrel{U_{m}}{\longrightarrow} \underset{1+T s}{\stackrel{k_{\mathrm{PWM}}}{1+T U_{a b}}}
$$

Fig.4 Inverter diagram

The block diagram of the closed-loop control system for inverter current inner ring is shown in fig.5.

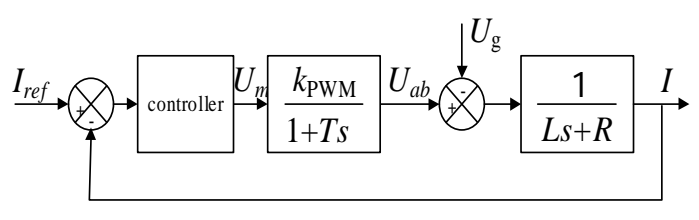

Fig.5 Current inner loop control diagram

\section{Reference Model Adaptive Control of the Current Inner Ring}

In Figure 5, $U_{g}$ is the grid voltage, and can be regarded as disturbance input. Let $U_{g}=0$, then open-loop transfer function of the controlled object is

$$
G_{\mathrm{p}}(s)=G_{1}(s) G_{2}(s) .
$$

When the inverter frequency is high enough, the switching cycle is small, $G_{2}(s) \approx k_{\mathrm{PWM}}$.

$$
G_{\mathrm{P}}(s)=k_{\mathrm{PWM}} \frac{1}{L s+R}=k_{\mathrm{p}} G(s) \text {. }
$$

When the command current amplitude, the inverter parameters and the filter inductance change with the operation of the system, $k_{\mathrm{PWM}}$ is slowly time-varying parameter. The adjustable gain model based on the MIT rate reference adaptive control can use to adjust controller parameters in real time.

The power electronics in the PWM inverter are switch elements, therefore the PV inverter system is a nonlinear system. If the higher harmonic is neglected, the system current inner loop transfer function can be obtained as shown in equation (6), which can reflect well the low frequency characteristic of the system. $G_{\mathrm{m}}(s)$ is selected as the system reference model, and let $G_{\mathrm{m}}(s)=k_{\mathrm{m}} \frac{1}{L s+R}=k_{\mathrm{m}} G(s)$, then the model reference adaptive control is established ${ }^{[10]}$ as shown in Fig.6. 


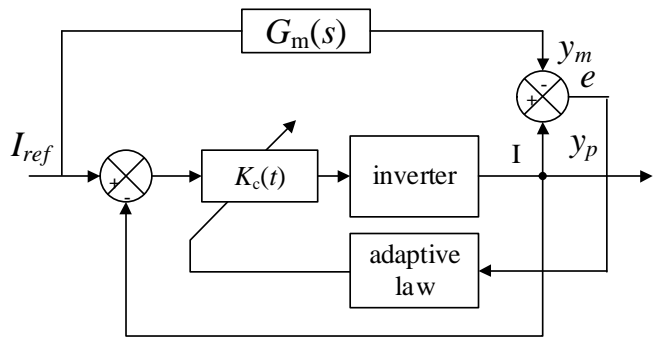

Fig.6 Adjustable gain MIT-MRAC system structure

Suppose the output of the system reference model is $y_{\mathrm{m}}$. The output of the controlled object is $y_{\mathrm{p}}$. The generalized error is defined as $e(t)=y_{\mathrm{m}}-y_{\mathrm{p}}$. The adjustable gain of MRAC is defined as $k_{\mathrm{c}}(t)$. When the performance index function $J=\frac{1}{2} e^{2}$ is introduced, adjustable gain along the negative gradient direction of $J$ according to the gradient-based MRAC ${ }^{[8]}$ is:

$$
\left.k_{\mathrm{d}} t\right)=-\gamma^{\prime} \frac{\partial J}{\partial k_{\mathrm{c}}}=-\gamma^{\prime} e \frac{\partial e}{\partial k_{\mathrm{c}}} \text {. }
$$

After derivation,

$$
k f(t)=\frac{\gamma}{k_{\mathrm{m}}} e(t) y_{\mathrm{m}}(t) \text {. }
$$

$\gamma=k_{\mathrm{p}} \gamma^{\prime}$ is the adaptive gain $(\gamma>0)$ informula (9). In order to avoid the closed-loop system divergence caused by the oversized input or $\gamma$ value being too large, the MIT normalization algorithm may be used to correct the adjustable gain ${ }^{[11]}$. Taken as

$$
\left.k_{\mathrm{c}} t\right)=-\gamma \frac{e \frac{\partial e}{\partial k_{\mathrm{c}}}}{\alpha+\left(\frac{\partial e}{\partial k_{\mathrm{c}}}\right)^{2}} \text {. }
$$

In the formula, $\alpha>0$, to avoid possible zeroing phenomenon. In order to avoid the adjustable gain is too large, a saturation characteristic can also be introduced to ensure that the parameter adjustment rate is always less than a given value. That is

$$
\phi(t)=-\gamma \mathrm{sat}\left(\frac{e \frac{\partial e}{\partial k_{\mathrm{c}}}}{\alpha+\left(\frac{\partial e}{\partial k_{\mathrm{c}}}\right)^{2}}, \beta\right) \text {. }
$$


In the formula, $\beta>0$, moreover, $\operatorname{sat}(x, \beta)=\left\{\begin{array}{cc}-\beta & x<-\beta \\ x & |x|<\beta \\ \beta & x>\beta\end{array}\right.$

After finishing:

$$
k f(t)=\gamma\left(\frac{e(t) y_{\mathrm{m}}(t) / k_{\mathrm{m}}}{\alpha+y_{m}^{2}(t) / k_{\mathrm{m}}^{2}}, \beta\right) \text {. }
$$

\section{Simulation Model}

In the simulation, the voltage at inverter DC side is $400 \mathrm{~V}$, the filter parameters are $L=2 \mathrm{mH}$, $R=1 \Omega$, and the grid voltage is $220 \mathrm{~V}$. In model reference adaptive control based on the MIT rate, the reference model $G_{\mathrm{m}}(s)=\frac{1}{0.002 s+0.1}$ is selected according to equation (7).

\section{MIT-based MRAC Control Simulation}

Building the simulation model in the Matlab/Simulink simulation environment shown in Figure 7, according to equation (9) to update the adjustable gain $k_{\mathrm{c}}(t)$, we can get the simulation results as shown in Figure 8.

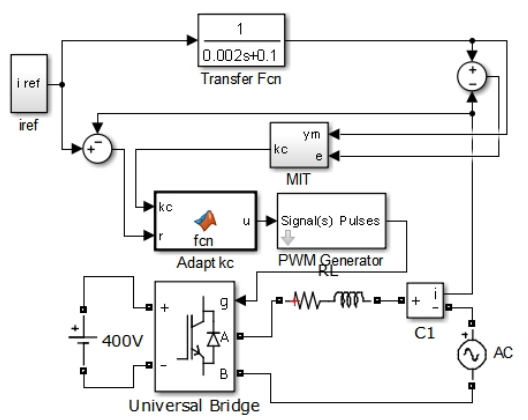

Fig.7 MRAC simulation model based on MIT
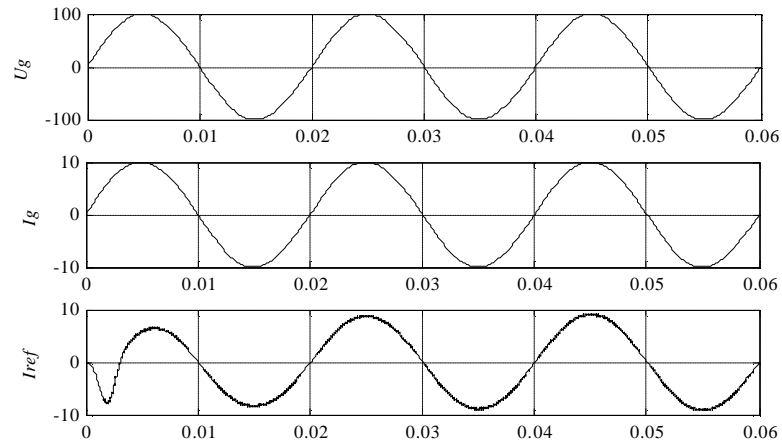

(a) Power grid voltage waveform, current instruction and actual waveform 

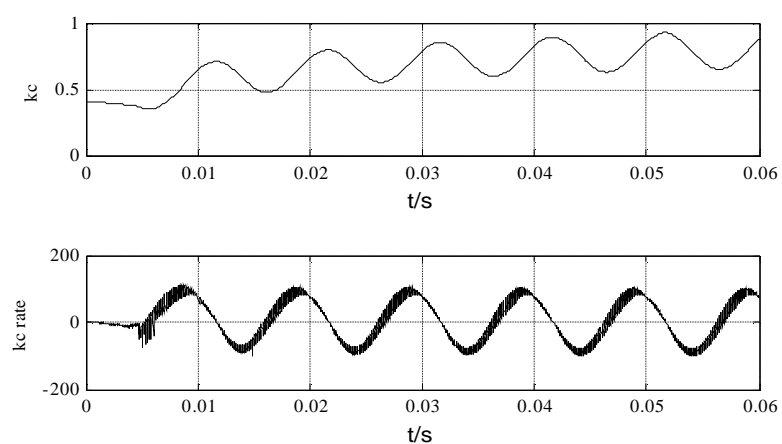

(b) Adjustment process of adjustable gain $k_{\mathrm{c}}(t)$

Fig. 8 Simulation waveform of MRAC control based on MIT

Figure 8 shows that the grid current can well track the phase of the command current using MIT-based MRAC control during $\gamma=5$. However, the input signal value or $\gamma$ is larger, the rate of change of the adjustable gain $k_{\mathrm{c}}(t)$ is larger and the waveform of grid current tracking command current is worsened near the amplitude of the current signal. The total harmonic distortion rate of the grid current is $4.61 \%$.

\section{Simulation of MRAC Control Based on Normalized MIT .}

In the above 3.1 simulation, the adjustable gain $k_{\mathrm{c}}(t)$ is updated according to the formula (11), and the MRAC based on the normalized MIT is performed. In the simulation, let $L=2 \mathrm{mH}, R=1 \Omega$, $\gamma=5, \alpha=0.01, L=2, \beta=2$. The simulation results are shown in Figure9.

As shown in fig. 9, the grid current can track the the reference current well adopting normalized MIT MRAC. When the magnitude of the command current changes from $50 \mathrm{~A}$ to $10 \mathrm{~A}$ at $0.06 \mathrm{~s}$, the grid current can also track quickly. At this moment, the total harmonic distortion rate of grid current is $0.79 \%$.
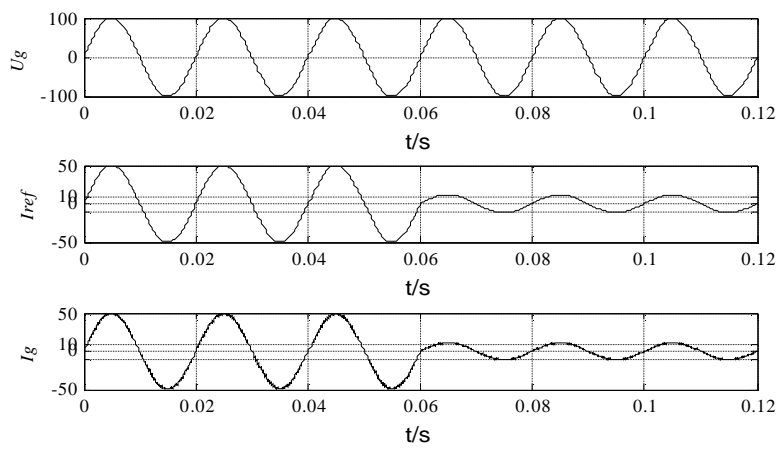

(a) Power grid voltage waveform, current instruction and actual waveform 

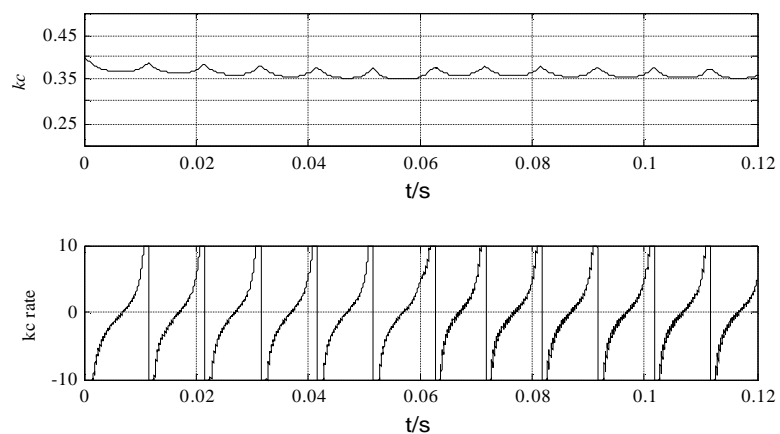

( b ) Adjustment process of adjustable gain $k_{\mathrm{c}}(t)$

Fig.9 MRAC simulation waveform based on normalized MIT

As shown in Fig.10, when $\gamma$ value is changed from 5 to 20, the change rate of adjustable gain $k_{\mathrm{c}}(t)$ is affected only, but the system follow-up control effect is good adopting normalized MIT MRAC. At this point, the total harmonic distortion of grid current is $0.83 \%$.
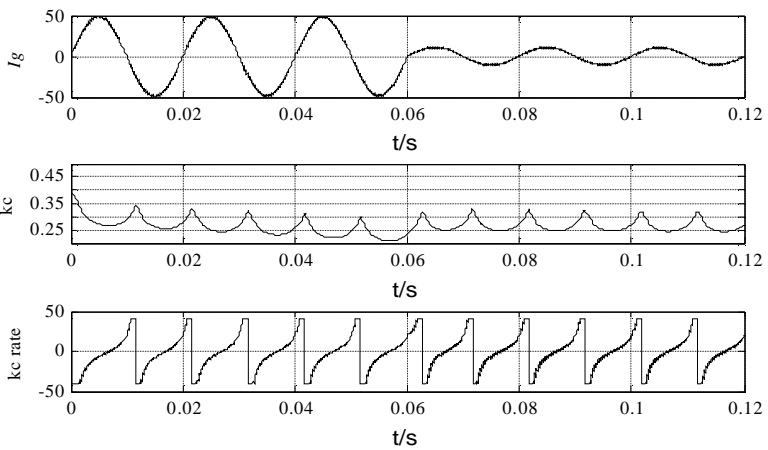

Fig.10 Simulation results during $\gamma=20$

As shown in Fig.11, When the AC side resistance of the inverter is changed from $R=1 \Omega$ to $0.01 \Omega$, the tracking effect of the grid current is good, and the total harmonic distortion rate of current is $0.63 \%$. When the AC side filter inductance is changed from $L=2 \mathrm{mH}$ to $1 \mathrm{mH}$, the tracking effect of the grid current is also good, the total harmonic distortion rate of current is $0.63 \%$.
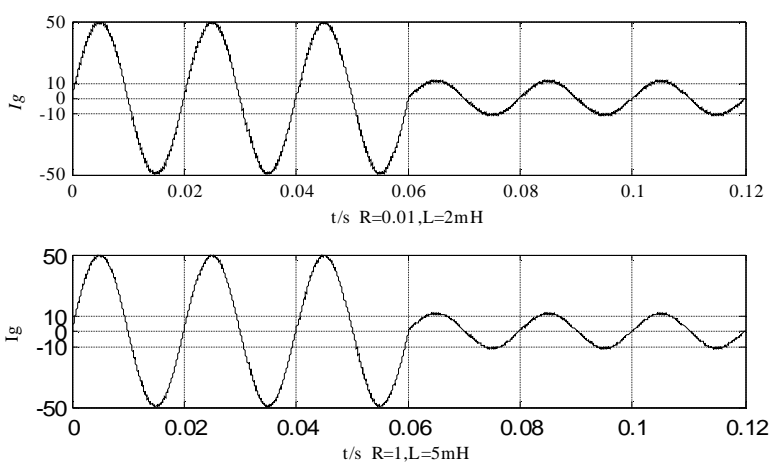

Fig.11 Simulation results of filter parameters change 


\section{Conclusion}

In this paper, the basic method of current inner loop control in single-phase PV grid-connected system is deeply studied, and a model reference adaptive control based on gradient method is proposed. The adjustable gain of MRAC is updated by using MIT and normalized MIT adaptive law respectively.The controller is simple in design and the parameter selection is not affected by the change of system parameters. The simulation results show that the controller parameters can be adjusted adaptively according to the changes of the filter parameters in the photovoltaic grid-connected system by using the normalized MIT adaptive law. The control effect is not affected by the adaptive gain and the input command current. The grid-connected current has good tracking effect on the command current, andfast tracking speed and strong robustness.

\section{References}

[1] XU Honghua. The study on development of PV technology in China[J]. Power System Technology, 2007, 31(20): 77-81.

[2] Zhou Lin, Feng Yu, Guo Ke, Liu Qiang, Jia Fangcheng, Huang Yong. Research on single-phasephotovoltaic grid-connected inverter modeling and control technology[J]. Journal of Solar Energy, 2012, 33 (03): 485-493.

[3] Zhao Zhengming and so on. Solar photovoltaic power generation and its application[M]. Beijing: Science Press, 2005.

[4] Khushboo Keshari,Satyanarayana Neeli, Vijayakumar K. Design of a sliding-mode-controlled dc-dc converter for MPPT in grid-connected PV System[C].International Conference on Electrical, Instrumentation and Communication Engineering (ICEICE2017),2017:842-850.

[5] Sina Vahid, Hassan Rastegar, S. Hamid Fathi.Improving the performance of PV grid interface inverter using the adaptive hysteresis band current control[C]. International Conference on Computer and Knowledge Engineering(ICCKE),2015:30-35.

[6] Gu Pan Pan, Zheng Jianyong, Miao Huiyu, Yang Jie, Yang Yun. A review of the research on grid inverter of photovoltaic power generation system[J]. Electrical Engineering, 2017, (11): 1-5 + 29.

[7] Zhang Xing, Wang Yangjun, Yu Changzhou, Qiao Caixia, Zhou Yanfeng, Ni Hua. Coupling mechanism and control strategy of grid-connected inverter with PI + repetitive control[J]. InState Electric Engineering, 2014,34 (30): 5287-5295.

[8] Chaojie He, Jinbin Zhao, Shuaitao Zhang, Shuuichi Ushiki. Analysis and implement of the single-phase voltage-controlled grid connected inverter[J]. IET Power Electronics, 2017, 10 (11): 1344-1352.

[9] M Swathi,P Ramesh.Modeling and Analysis of Model Reference Adaptive Control by Using MIT and Modified MIT Rule for Speed Control of DC Motor[C].IEEE 7th International Advance Computing Conference,2017:482-486.

[10]Chai Tianyou, Yue Heng with. Adaptive Control[M]. Beijing: Tsinghua University Press, 2016.

[11] Pang Zhonghua, Cui Hong. Editing system identification and adaptive control MATLAB simulation[M]. Beijing: University of Aeronautics and Astronautics Press, 2013. 